\title{
Thread-based wearable devices
}

\author{
Junfei Xia $\odot$, Shirin Khaliliazar, Mahiar Max Hamedi, ${ }^{*}$ and \\ Sameer Sonkusale* ${ }^{*}$
}

\begin{abstract}
One-dimensional substrates such as textile fibers and threads offer an excellent opportunity to realize sensors, actuators, energy harvesters/storage, microfluidics, and advanced therapies. A new generation of wearable devices made from smart threads offer ultimate flexibility and seamless integration with the human body and the garments that adorn them. This article reviews the state of the art in thread-based wearable devices for monitoring human activity and performance, diagnoses and manages medical conditions, and provides new and improved human-machine interfaces. In the area of new and improved human-machine interfaces, it discusses novel computing platforms enabled using thread-based electronics and batteries/ capacitors. For physical activity monitoring, a review of wearable devices using strain sensing threads is provided. Thread-based devices that can monitor health from biological fluids such as total analysis systems, wearable sweat sensing patches, and smart sutures/smart bandages are also included. The article concludes with an outlook on how fibers and threads are expected to impact and revolutionize the next generation of wearable devices. Knowledge gaps and emerging opportunities are presented.
\end{abstract}

\section{Introduction}

Recent technological advances in smart flexible materials, processing and fabrication have progressively changed the landscape of healthcare and wellness toward wearable platforms for real-time monitoring and treatment. ${ }^{1-5}$ Flexibility, conformability, and stretchability are desirable virtues in such platforms. There are many examples from flexible electrodes for electrocardiogram (ECG) or electroencephalogram (EEG) monitoring; wearable physical sensors for monitoring body temperature, humidity, touch/pressure, and biochemical sensors for monitoring $\mathrm{pH}$, electrolyte and metabolite levels in sweat and other body fluids for real-time health monitoring. ${ }^{6-12}$ These examples also include flexible transistors and batteries to power these devices for a selfcontained flexible bioelectronic platform. Materials such as polyimide ${ }^{13}$ and parylene ${ }^{14}$ have been used as substrates for such flexible bioelectronics. However, making these devices is still expensive because it requires clean room facilities for top-down lithographic patterning. Advances in screen printing and inkjet printing have partially mitigated some of these concerns. It must be noted that existing flexible devices have essentially remained two-dimensional (2D) and conform to the skin or scalp. It is extremely challenging for these platforms to adapt to the complex three-dimensional (3D) contours of the human body for the next generation of wearable devices. Most organs and tissues are 3D heterogeneous multilayered biological structures. It would be a significant advance if one could integrate functional components, such as sensors and electronics, in a way that can adapt to such 3D surfaces. For example, wounds and fractures, which have complex 3D structures, would greatly benefit from physical (e.g., strain) and chemical (e.g., pH) sensors that can monitor the tissue/organ environment and provide valuable information to optimize patient-specific treatments.

This brings us to textiles and threads/yarns as possible substrate for the next generation of flexible bioelectronics. Advanced thread/yarn manufacturing and textile-making provides a relatively inexpensive route to make smart thread-based wearable platforms through relatively simple modifications to the production and processing of fiber materials. This has led to thread as a convenient multifunctional substrate for applications ranging from sensing to tissue

\footnotetext{
Junfei Xia, Department of Electrical and Computer Engineering, and Nano Lab, Advanced Technology Laboratory, Tufts University, USA; junfei.xia@tufts.edu

Shirin Khaliliazar, Department of Fibre and Polymer Technology, School of Engineering Sciences in Chemistry, Biotechnology and Health, KTH Royal Institute of Technology, Sweden; shirink@kth.se

Mahiar Hamedi, Department of Fibre and Polymer Technology, School of Engineering Sciences in Chemistry, Biotechnology and Health, KTH Royal Institute of Technology, Sweden; mahiar@kth.se

Sameer Sonkusale, Department of Electrical and Computer Engineering, and Nano Lab, Advanced Technology Laboratory, Tufts University, USA; sameer@ece.tufts.edu *Corresponding author

doi:10.1557/s43577-021-00116-1
} 
engineering to diagnostics. Electrically conductive threads can be categorized into three groups: (i) metallic threads, like thin metal- coated threads including silver and goldcoated threads, (ii) semiconductive threads made of conducting polymer-coated fibers and carbon-based materials such as carbon nanotubes (CNTs), graphene, or carbon fibers, and (iii) composite materials which are the combination of electrically conductive and non-conductive $2 \mathrm{D}$ or $3 \mathrm{D}$ - materials such as nanoparticles in fiber-based networks. ${ }^{15}$

Some key merits of threads and textile substrate that make it truly remarkable are the diversity of thread options available from elastic threads to biodegradable threads, its hierarchical nature, potential for physical and chemical modification, and ability to be integrated into textiles using two methods: one is additive, where threads are either embroidered into textile or active materials screen-printed onto textile. The other is a bottom-up approach, where threads used as for certain basic functions like sensing, are woven or knitted to create textile-based wearable analytical systems via sewing or suturing. This offers a robust and cost-effect approach for the fabrication of thread-based devices. Thus, threads and textiles are uniquely positioned as a substrate to exist as a standalone device, or as the whole platform itself for the next generation of wearables. ${ }^{16}$

\section{Thread and textile-based wearable computing}

Wearable devices are particularly interesting if they can integrate electronic components, such as wires, transistors, and electronic-sensing modalities and connect to the cloud, to enable a digital wearable micrototal analysis system ( $\mu$ TAS) for medical and consumer electronics application.

In general, the realization of true textile-based components requires individual threads that are different in form and function to realize allthread-based wearable computing. A key component is an electrically conductive thread as a basic circuit element used to realize wires, electrodes, interconnects, and antennas. There are several different threads available commercially, as well as laboratory scale, to meet this need that enable endless possibilities.

The major categories of such electroactive threads comprise:
1. Metallic threads: these threads can be either coated with thin metals (such as gold and silver) or comprise pure metallic multifilaments (e.g., stainless steel filaments mixed with polymer fibers forming threads). These threads are particularly interesting as wires for electronic connections, as parts of electronic components, or as electrodes in electroanalytical devices (particularly inert metals such as gold).

2. Semiconductive threads: the most versatile materials here are conducting polymers and carbon nanomaterials (CNTs, graphene, carbon fibers $)^{17}$ because these materials, in contrary to solid-state semiconductors, can be coated onto threads or in some cases, extruded into fibers. The use of semiconductive materials enable threadbased transistors or optoelectronic devices. Carbonbased materials are interesting for both energy storage devices ${ }^{18}$ and electroanalytical systems.

3. Composite materials: The combination of many materials such as nanoparticles and an ever-increasing range of 2D materials are enabling many new forms of composite materials that can be combined with the other threads especially for energy storage.

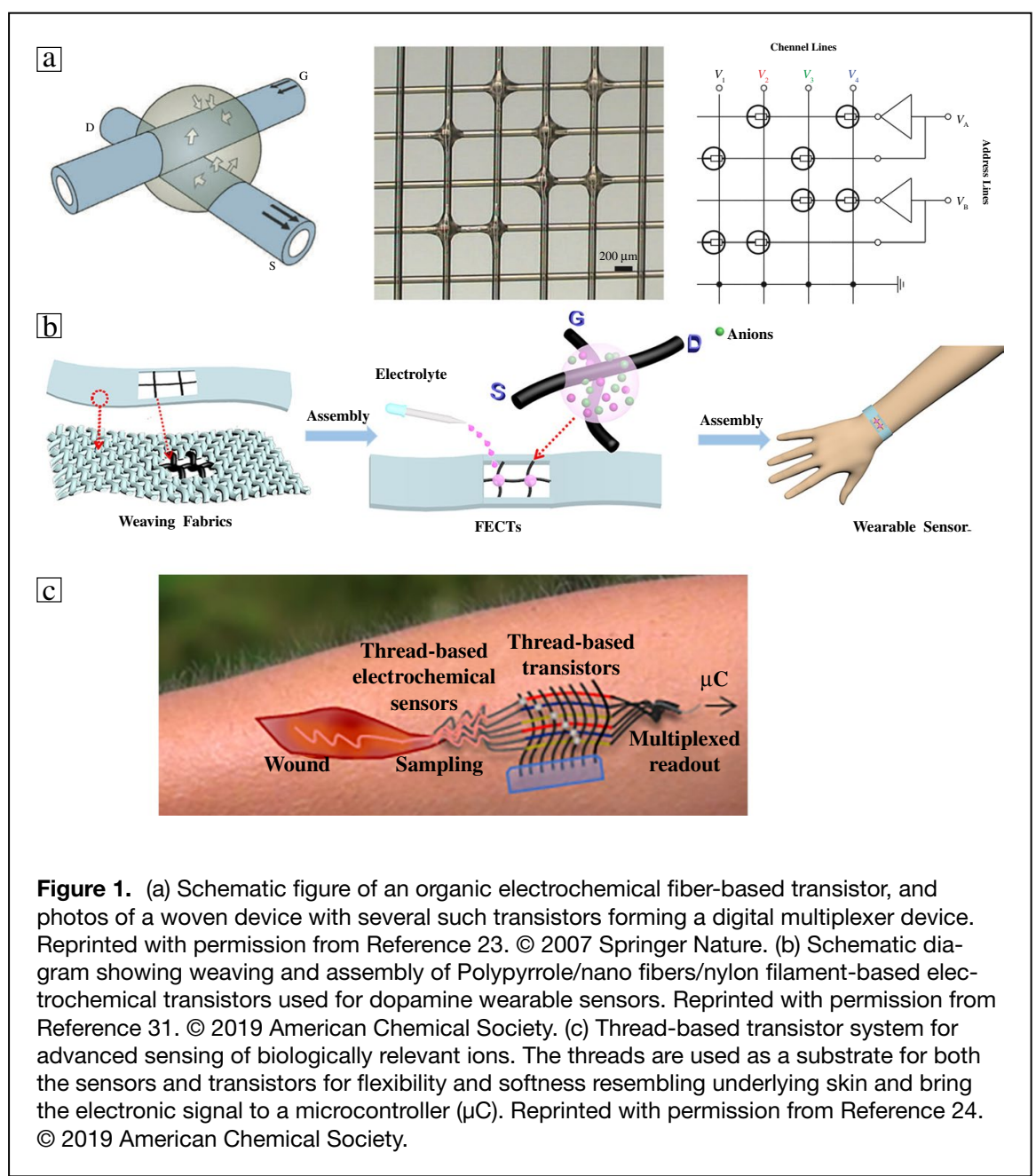




\section{Transistors and electronics}

The realization of thread-based transistors is interesting because transistors are the basic element in all digital logic, thereby enabling woven/textile logic, and also because electrolyte gated transistors can be used as sensing elements in analytical devices.

A few early organic field effect transistors were realized on fibers, ${ }^{19-21}$ but these devices required thin dielectric gates that were not compatible with the 3D geometries of textiles and did not enable electroanalytical systems. These problems were addressed by the introduction of thread-based organic electrochemical transistors (OECTs) demonstrated by Hamedi et. al (Figure 1a-b) ${ }^{22,23}$ These transistors rely on conducting polymers, usually coated on threads, and connected via an electrolyte to other threads to form the channel, which changes conductivity upon redox reactions.

Thread-based transistors have since then been used in numerous applications for wearable devices (Figure 1c). ${ }^{24}$ Natural and synthetic fibers such as cotton, silk, and nylon, are used for the fabrication of flexible fiber organic electrochemical transistors (FECTs). These fibers become electrically conductive by deposition of a thin layer of metals/conductive polymers on their surfaces. Battista et al. fabricated an integrated fiber-based OECT using PEDOT:PSS-coated cotton yarns decorated by noncovalently adsorbed fungal laccase POXA1b for direct detection of Tyrosine (L-Tyr)
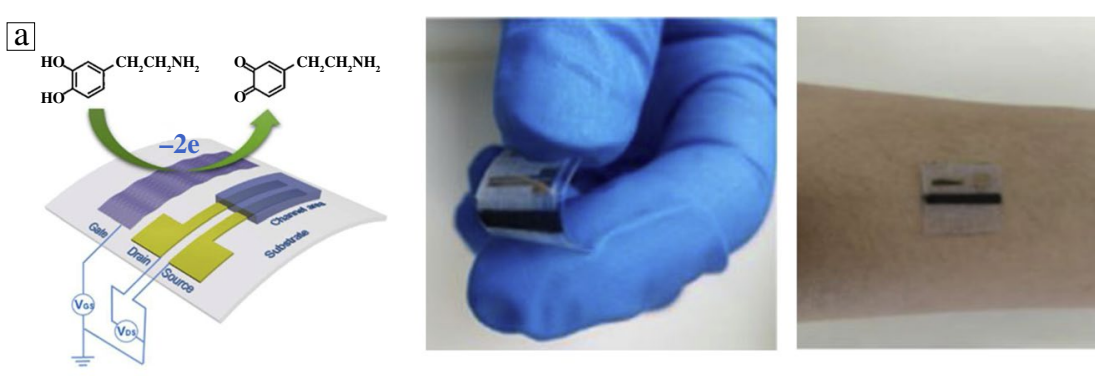

b

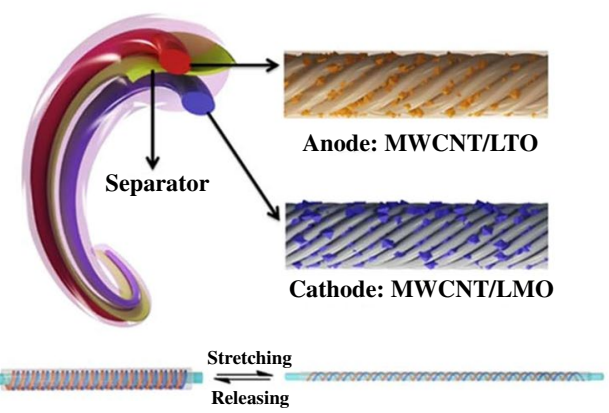

Figure 2. (a) (Left) Schematic illustration of A Nafion/reduced graphene oxide (rGO)/carbonization of a silk fabric (CSF) organic electrochemical transistor (OECT) sensors based on fabrics and flexible sheets. Electro-oxidation of dopamine on the gate electrode enables the sensing (right) photos of the flexible device on skin. Reprinted with permission from Reference 28. (C) 2020 Elsevier. (b) Schematics of a flexible wire-shaped lithium-ion battery using woven yarns of active materials, and the fiber-shaped lithium-ion batteries directly woven into flexible textiles. Reprinted with permission from Reference 34. (C) 2014 Wiley. Note: MWCNT, multiwalled carbon nanotube; $\mathrm{LTO}, \mathrm{Li}_{4} \mathrm{Ti}_{5} \mathrm{O}_{12}$; $\mathrm{LMO}, \mathrm{LiMn}_{2} \mathrm{O}_{4}$. amino acid in human physiological fluids. This textile-based biosensor monitored the biomarker through a mediator-less catalytic reaction. ${ }^{25}$ Yang et al. developed a multilayer-modified fiber electrode using $\mathrm{Cr} / \mathrm{Au} / \mathrm{PEDOT}$ :PSS core-shell structure on nylon (PA6) fibers. The biosensor showed stable performance at bending tests when it was woven into a diaper to detect glucose in artificial urine. ${ }^{26}$

Carbon materials are often considered as an alternative to metal electrodes due to their low cost, wide potential window, and relatively inert electrochemistry. ${ }^{27} \mathrm{Ji}$ et al. proposed a noble metal-free gate electrode for fabric-based OECT by carbonization of a silk fabric (CSF) followed by the surface modification with reduced graphene oxide (rGO) and Nafion (Figure 2a). The hierarchical structure of CSF improved the dispersity of rGO and Nafion on the fabric surface, which resulted in conductive and flexible fiber electrodes comparable to metal gate electrodes in terms of sensitivity and selectivity for detecting dopamine in artificial urine. ${ }^{28}$ Modification of nylon (PA6) fibers with a composition of multiwalled-carbon nanotubes (MWCNTs) and polypyrrole (PPy) conducting polymer offered a fiber-based OECT with a higher on/off ratio, better stability, and greater flexibility in comparison with PPycoated nylon (PA6) fiber electrode. The presence of MWCNTs led to the growth of a large area of the PPy nanowires that improved the morphological and electrical properties of the biosensor. $^{29}$

Introducing polymeric nanofibers (NFs) to the surface of fibers is a novel approach to enhance the specific surface area of the fiber electrodes and improve the hydrophilic properties of the synthetic ones. ${ }^{30}$ Qing et al. prepared a poly(vinyl alcohol) (PVA)-co-polyethylene (PE) nanofibers coated nylon (PA6) filaments and in situ polymerized PPy on the surface of the filaments. The PVA-co-PE nanofibers on the surface facilitate the formation of an interconnected conducting PPy network. ${ }^{31}$

Recently, a new class of threadbased transistors (TBTs) were introduced that use electrolyte gating of organic and inorganic semiconducting channel coated threads. ${ }^{24}$ The novelty is in the use of nonvolatile room-temperature gels of ionic liquids (or ionogels) serving as an allaround electrolyte gate dielectric. Drain and source contacts are made through textile knotting of metal wire on either end of thread channel, and a metallic thread/wire serves as gate. Both poly(3-hexylthiophene-2,5-diyl) (P3HT) and CNTs were explored as 
semiconducting channel. The transistors were used to realize logic gates and also served as an analog multiplexer in a multiplexed diagnostic application where TBT was connected to thread-based electrochemical sensors for tissue embedded diagnostics. Since knotting is not scalable, a new approach for 3D stencil-based patterning of source, drain and gap region on thread has also been proposed that allows parallel fabrication of tens or hundreds of transistors. ${ }^{32}$

\section{Batteries and supercapacitors}

Batteries and supercapacitors were initially developed in sandwiched planar thin films and jelly roll designs, which are available in various standards. The emergence of wearable devices together with the rapid development of new materials for supercapacitors and Li-ion batteries has, however, resulted in the demonstration of various types of energy storage devices that are safer and flexible for additive integration. These types of flexible batteries are already being commercialized by several companies. ${ }^{33}$ A more interesting approach for future wearables will rely on energy storage devices that are integrated at the thread/fiber level of the textile to enable energy storage in textile wearables. The direct integration of batteries and supercapacitors on the fiber level can enable both the flexibility from the fiber itself and the inherent stretchability of textiles. It further enables direct integration into the textile without comprising comfort (Figure 2b). ${ }^{34}$ These developments are enabling a range of advanced fiber-based batteries and supercapacitors; this topic is reviewed in detail elsewhere. ${ }^{35}$ The seamless integration of textile-based sensors and textile-based energy storage devices will enable energy autonomous wearables. ${ }^{36}$

\section{Physical activity monitoring}

Threads are ideal materials for making wearable strain and pressure sensors as they can be directly sewn into existing clothing or create separate patches to use as standalone sensors. The mechanical properties of threads such as the ability to bend, twist, and stretch make them naturally suitable as physical sensors. While the mechanism for sensing varies depending on the material and the application, all thread-based physical sensors use conductive threads or yarns. Intrinsically conductive threads are favored by many researchers due to their high conductivity. Alternatively, non-conductive threads can be coated with conductive inks as mentioned previously. The coating offers great tunability of properties of the threads and could play an important role in the sensor's performance.

\section{Strain and pressure sensing threads}

Thread-based strain and pressure sensors can be classified into two types: resistive based and capacitive based. In a resistivebased sensor, the stretching changes the resistance of the thread resulting from changing in its length and geometry. In a capacitive-based sensor, two conductive threads are separated by a layer of dielectric material to form a capacitor. When stretched or compressed, the deformation changes the dimension of the device, which results in the change of device capacitance. Cheng et al. fabricated a graphite-based sensor using elastic threads as the base material. The elastic thread composed of a polyurethane fiber (stretchable) and polyester fibers wound helically (Figure 3a). ${ }^{37}$ In another work by Sadeqi et al., polybutylene terephthalate puffy (PTB) threads were used as the substrates for building strain sensors and the resistance was measured as a function of applied strain (Figure 3b) ${ }^{38}$ Metallic nanowires, including gold and silver nanowires, are used to functionalize threads and provide them with excellent electrical and mechanical properties for strain sensing applications (Figure $3 \mathrm{c}) .{ }^{39}$ A capacitive-based pressure sensor can be realized using composite yarns. The capacitive sensor unit was formed by conductive Ni-coated cotton yarn electrodes as the core and the nanofiber dielectric layer coated as the shell (Figure 3d). ${ }^{40}$ Pressure sensing threads have also been utilized in human gait monitoring, which provides indications of a person's health conditions. Alaimo et al. reported a smart insole for gait monitoring enabled by embedding strain sensing threads into a shoe insole (Figure 3e). The conductive threads were arranged in a 2D grid format for mapping foot pressure and cast in a highly elastomeric EcoFlex sole. The sensor can accurately map foot pressure during gait cycles as well as detect an eventual anomaly during the user's gait. ${ }^{41}$

\section{Wearable diagnostics}

Wearable diagnostic platforms are integrated systems with a collection of sensors, electronic circuitry, and communication infrastructure. As discussed, threads provide an ultimate toolkit to realize sensors, electronics, microfluidics, conductors, interconnects, and antennas to realize an all-thread-based platform. In this section, we will highlight some of these exquisite thread-based platforms ranging from a micro-total analysis system or $\mu$ TAS to analyze biomolecules of interest, sweat sensing patches to monitor biomarkers of fatigue and exhaustion, smart sutures and smart bandages that can monitor chronic wound, and even pollution-detecting gas sensing textiles.

\section{Total analysis systems}

Cellulose substrates, including paper, cloth, and thread are the most attractive materials for fabricating wearable microfluidic analytical devices due to their micrometer-scaled fluidic structure. $^{42}$ This structure enables passive fluid flow, driven by capillary forces through fiber-based microchannels, which eliminates the need for external pressure pumps to guide the fluid into microfluidic channels. Besides that, cellulose substrates are lightweight, flexible, durable, biocompatible, and importantly, the large surface-to-volume ratio of the network of cellulose fibers enables integration of electrodes and electroanalytical sensors in their substrates and allows immobilizations of reagents to perform further analytical assays. ${ }^{43}$ Cloths and threads gained lots of attention for fabricating wearable 


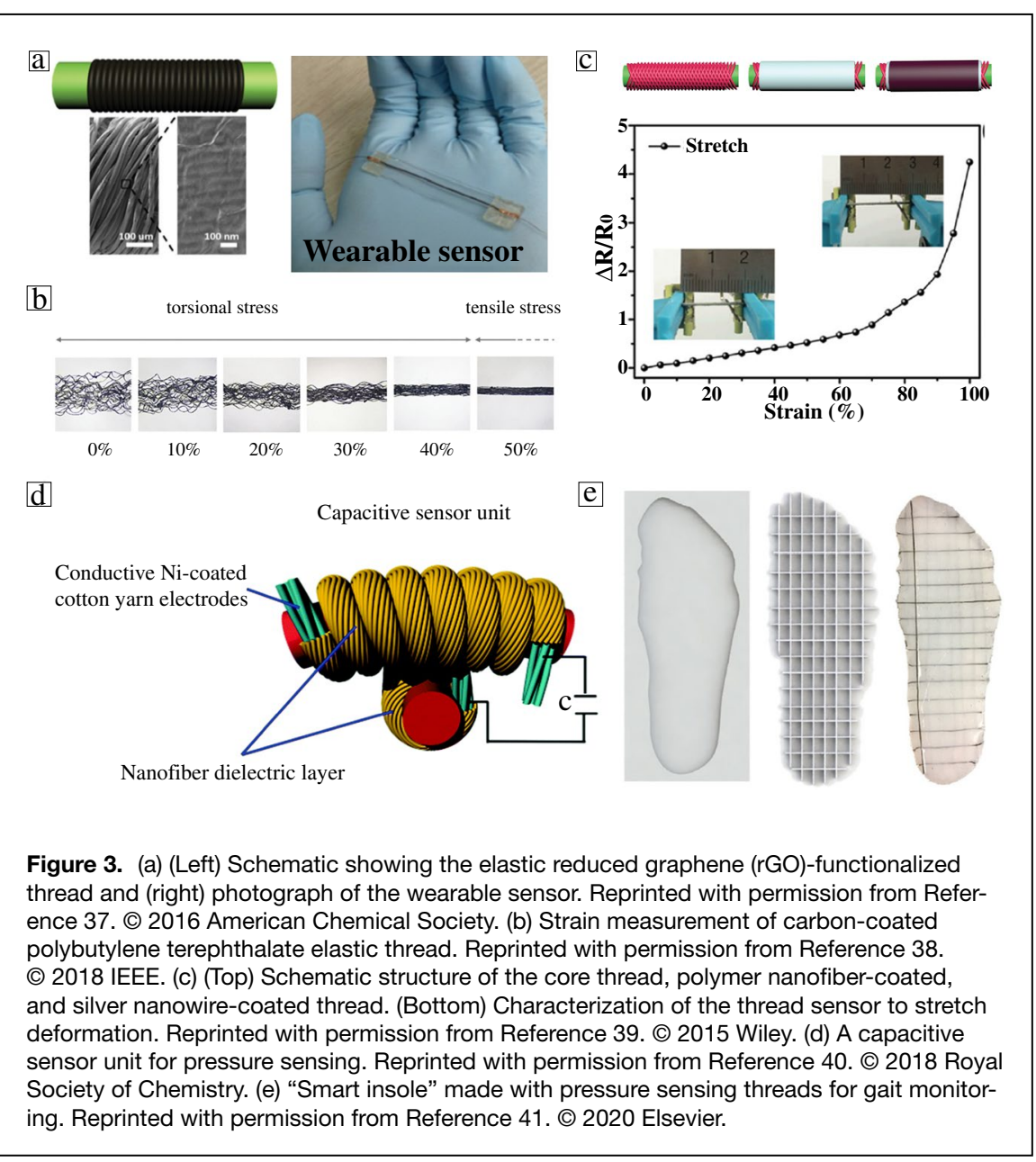

biosensors because they show higher mechanical strength, flexibility, and durability in wet conditions and because the structure of fibers have inherent 3D microchannels for microfluidics. There is also a wide selection of available natural and synthetic threads that can be functionalized to incorporate electronics for the development of electronic biosensors. ${ }^{44,45}$

There are two principal textile manufacturing methods for the integration of these functions: the first is additive fabrication such as printing on textiles or embroidering threads into textiles to add function (Figure $4 \mathrm{a}-\mathrm{b})^{45,46}$ and the second is a bottom-up approach where all base functions are embedded at the fiber level, and weaving or knitting is used to design and create the $\mu$ TAS (Figure 4c) ${ }^{47}$

Although both of these fabrication approaches offer low cost and robust wearable biosensors without the need for undergoing complicated microfabrication processes at cleanrooms, the first approaches, including screen-printing ${ }^{48}$ and embroidery techniques, ${ }^{46,49}$ are widely used due to their potential of scalability, and therefore, are amenable to industrial applicability.

Kinnamon et al. fabricated a textile screen-printed biosensor to detect the influenza A virus (Figure $4 b$ ). To fabricate electrodes for the electrochemical textile biosensor, conductive silver ink was first screen-printed onto two different flexible substrates, including a nonporous polyamide sheet and a commercially available utility textile. Second, the graphene oxide (GO) layer was printed in a complementary pattern to the printed silver electrode and was functionalized with an influenza-specific affinity assay. This electrochemical sensor showed more stable and reproducible responses when printed on a textile substrate compared to the polyamide sheets. ${ }^{50}$ Gualandi et al. used a single-step screening technique to develop a fully textile-based organic electrochemical transistor (OCET) using PEDOT:PSS conductive polymer. The proposed wearable chemical sensor showed reliable results as a textile-based transistor to develop chemical sensors for realtime monitoring of redox-active biomolecules such as dopamine, ascorbic acid, and adrenalin. ${ }^{51}$

The machine embroidery method facilitates the rapid integration of conductive threads in customized geometries and configuration into textile substrates, which leads to the fabrication of mechanically robust biosensors with mass-production capability. ${ }^{52} \mathrm{Liu}$ and Lillehoj fabricated an embroidered electrochemical sensor on a cotton t-shirt for monitoring glucose and lactate in buffer and whole blood samples. Carbon-coated polyester yarns were modified with enzyme probes and embroidered into a polyester fabric along with silver/silver-chloride coated polyester yarn and carbon-coated ones as reference and counter electrodes, respectively. The fabricated biosensor showed reproducible results with proper mechanical properties for multiplexed measurements of the biomolecules. ${ }^{53} \mathrm{~A}$ wearable electronic nose (e-nose) constructed as an embroidered gas sensor on polymer/functionalized single-wall carbon nanotubes (SWCNTs) nanocomposites. These sensing materials were coated onto already embroidered conductive threads on a fabric substrate. The fabric-based e-nose could discriminate between the body odor of two people and detect body odors in different forms such as urine, armpit, and exhaled breath odor using simple recognizing algorithms. ${ }^{54}$

Although the bottom-up fabrication approach has attracted less attention, it allows complex designs and multiplexed detection of analytes and is free of patterning and related alignment issues. This approach was used to develop a woven 


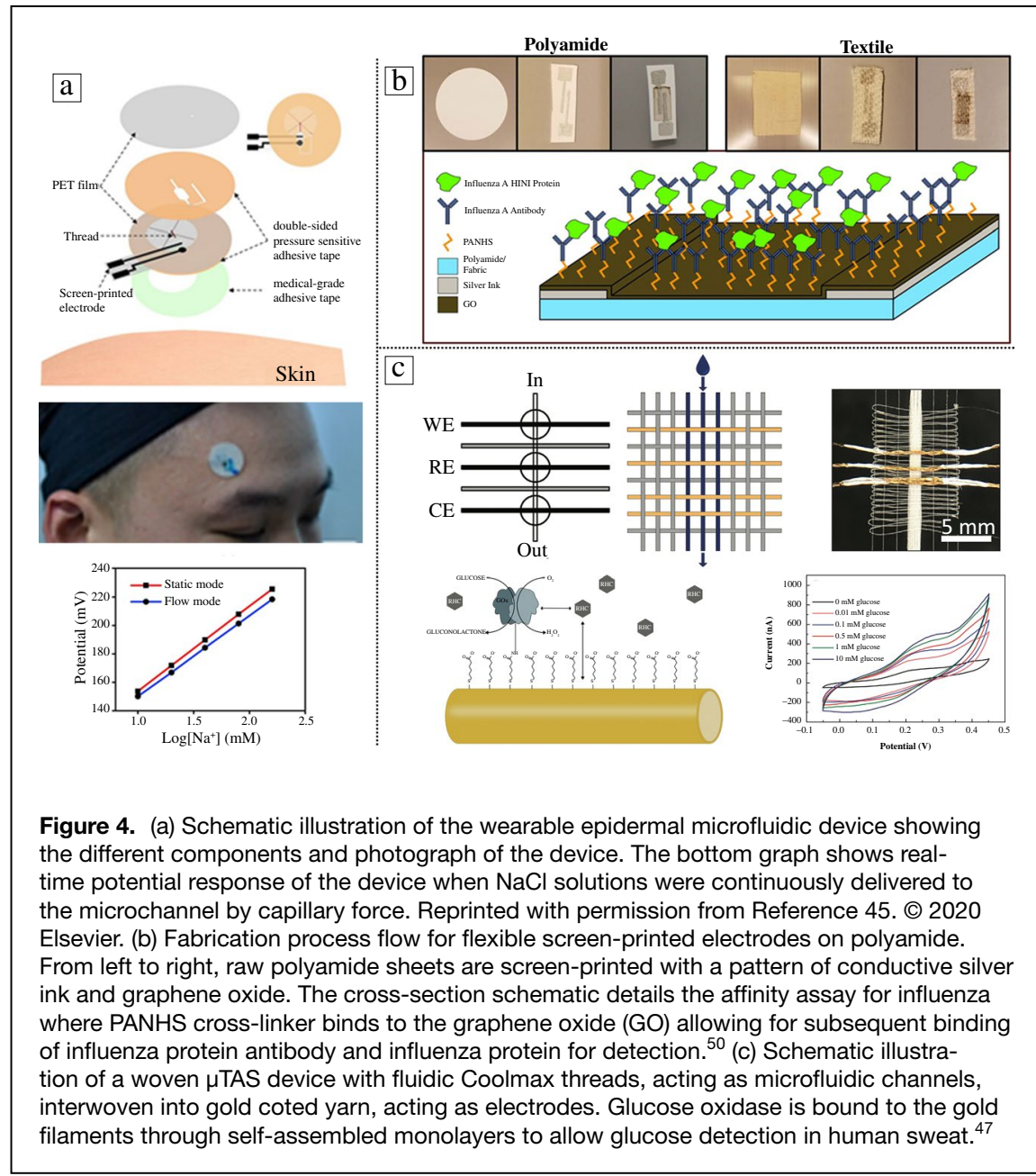

biosensor in which the test area was defined by weaving gummed and degummed silk yarns as hydrophobic barriers and hydrophilic substrates. The developed electrochemical patch sensor showed analogous electrochemical behavior compared to screen-printed counterparts. ${ }^{55}$

\section{Sweat-sensing patch}

Wearable sweat sensors provide a real-time and quantitative analysis of sweat components such as electrolytes and metabolites, which can be used as biomarkers for disease diagnosis. Being continuously secreted by the body, sweat can be easily collected and is one of the most accessible body fluids for onbody measurement. Thread-based wearable sweat sensors have been developed over the years with several promising prototypes capable of continuous, real-time, and sensitive sweat sensing without compromising wearer's comfortableness. Threads are made from tens to hundreds of twisted fibers that are packed in a cohesive bundle. The natural capillary action in threads provides passive wicking and sampling of sweat. Owning to their high absorbability, mechanical durability, and ultimate flexibility, threads are ideal substrates for wearable electrochemical sensors that provide ultrafast and highly sensitive detection. Individual thread electrodes can be assembled into a patch format for conformal skin contact using weaving, knitting, or embroidering techniques.

As with physical sensors, a conductive coating is required for threads that are subsequently functionalized with enzymes or ion-selective membranes for detection of biomarkers in the sweat such as lactate or electrolytes (Figure 5a). Modali et al. developed a thread-based wearable patch sensor for sensing sweat lactate. Nylon yarns were sequentially coated with carbon ink and lactate oxidase (LOx). LOx selectively converts lactate into pyruvate and hydrogen peroxide. The oxidation current was measured as a function of lactate concentration. The patch was fabricated using textile weaving technology with high scalability (Figure 5b). ${ }^{55,56}$ Similarly, MWCNT and PPy-modified nylon fibers were assembled into a fiber-based OECT for sweat lactate sensing. ${ }^{29}$ In another work, multiplexed sweat sensing threads were integrated into a commercial bandage as shown in Figure 5c. ${ }^{57}$ Apart from sweat biomarkers, the perspiration level is another important indicator of one's physical status and comfort level. Conductive threads were packed inside a cotton cover to sample sweat where the change in resistivity was measured as a function of perspiration level (Figure 5d). The sensor can be sewed into multiple locations of a shirt for onbody, real-time sweat monitoring. ${ }^{58}$

\section{Smart sutures/smart bandages}

Thread-based sensing and delivery platforms can be used to realize the so-called "smart suture" for wound monitoring and therapy. The flexible nature and widely available selection of thread materials make them suitable as sutures where high mechanical strength and tissue compatibility are required. In addition, the functionalization of threads renders them capabilities of sensing and drug delivery that are not achieved by conventional sutures. Early work in this field includes coating surgical sutures with antibiotics to prevent wound infection, as well as sutures made from shape-memory polymer for temperature-controlled wound closure. ${ }^{59}$ In recent years, there is a growing trend for incorporating hierarchical structures and novel multifunctional materials into sutures to achieve improved performance in vivo. 
Liu et al. developed a biomimicking sensing suture (BBS) for simultaneous wound monitoring, wound healing and antibacterial activity. Inspired by spider-silk fiber, the authors fabricated core-shell multilayered sutures where the silk-based core fiber provided a mechanical framework of the suture and the three layers in the shell consisted of a conductive CNT for strain sensing, growth factors for in situ delivery, and quantum dots for optical monitoring of drug release (Figure 6a). ${ }^{60}$ In another pioneering study by Wang et al., functionalized MWCNTs were synthesized and twisted into helical fibers as implantable sensors. The sensing ability was achieved by functionalizing a CNT with different sensing molecules. Due to the small size and mechanical stability offered by the hierarchical helical structure, the fiber-based sensor can be implanted either to a tumor site or blood vessel simply with a syringe needle (Figure 6b). Such fiber-based devices can be potentially used as sutures for wound monitoring due to their small size and the multiplexed sensing capability. ${ }^{61}$

On-demand drug delivery to the wound site is of crucial importance for wound healing. Traditional wound dressing uses passive drug release that lack both spatial and temporal control over the dosage. Smart wound dressing that is capable of on-demand drug delivery triggered by either internal stimuli such as $\mathrm{pH}$ or temperature change at the wound bed or external stimuli, including temperature, ultrasound, electrical, and magnetic fields would significantly improve the outcome of wound healing. Mostafalu et al. developed a threadbased smart bandage for controlled wound drug delivery with enhanced spatiotemporal resolution. ${ }^{62}$ Composite threads were fabricated with a hydrogel coating containing thermoresponsive drug carriers. Each thread functioning as an independent heating unit could be individually triggered for on-demand drug release (Figure 6c).

\section{Gas-sensing threads}

Textile-based gas sensors that are lightweight, wearable, and low cost provide real-time environmental monitoring and detection of human health. For example, threads-based gas sensors can be easily integrated into clothing for monitoring toxic and corrosive gases in the work places. Compared with planner substrate such as paper for gas sensing, threads have high surface area to volume ratio for achieving high sensitivity. Optical and electronic detection are the two major types of sensing mechanism employed in thread-based gas sensor. In optical approaches, threads are functionalized with gas-sensitive dyes that change their optical properties upon interacting with the gas species. The colorimetric method stands out for equipment-free readout. In electronic approaches, threads are made conductive and their electrical properties such as resistance changes with gas absorption.

Owyeung et al. fabricated washable gas sensing threads by entrapping $\mathrm{pH}$ indicators and a metalloporphyrin dye into the threads. A poly(dimethylsiloxane) (PDMS) coating was developed to prevent dye leaching for repeated use cycles (Figure 7a). ${ }^{63}$ In another colorimetric gas sensor, electrospun nanofiber yarn was loaded with lead acetate and lead iodide for sensing breath biomarker hydrogen sulfide and hazardous ammonia gas (Figure 7b). ${ }^{64}$ Note that ammonia is also a breath biomarker associated with kidney malfunction. ${ }^{65} \mathrm{Col}-$ orimetric dyes were incorporated into yarns by adding them in the electrospinning solutions. Ionic liquid was doped into nanofibers as an absorbent material for gases and was shown to improve the sensing performance. Apart from colorimetric sensing, Seesaard et al. have developed a fabric-based chemical gas sensor for human odor monitoring by drop casting carboxylic-functionalized single walled carbon nanotube (SWCNT-COOH)/polymer solution onto conductive threads. The electrical resistance of threads changed upon adsorption of gas species (Figure 7c). ${ }^{54}$

b

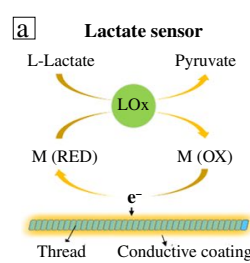

c
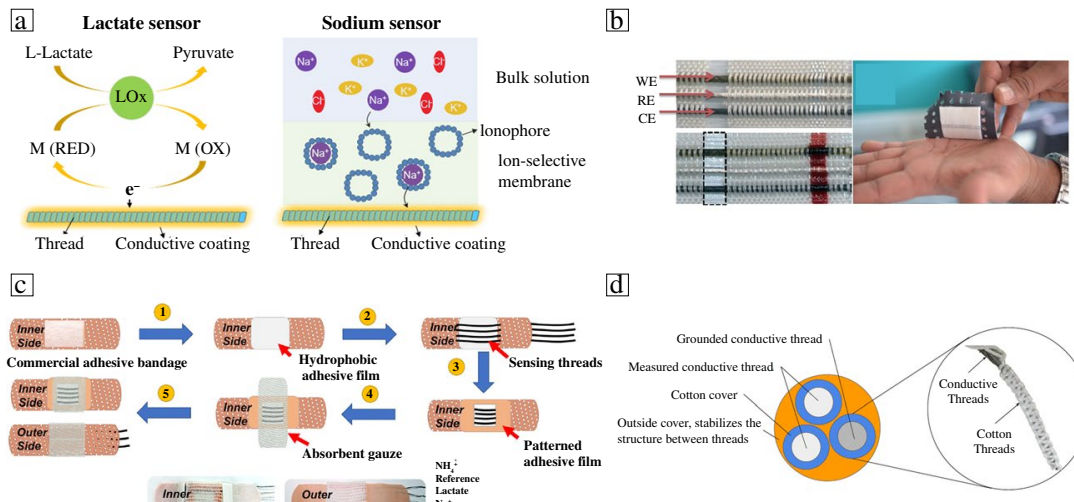

d]
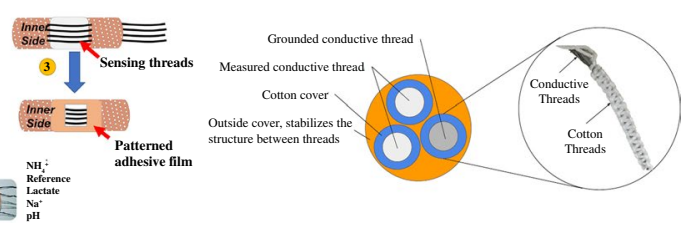

Figure 5. (a) Schematic illustration of thread-based lactate sensor and sodium sensor. (b) Thread-based sweat sensing patch for lactate detection. Reprinted with permission from Reference 55. (C) 2015 Wiley. Reprinted with permission from Reference 56. () 2016 Royal Society of Chemistry. (c) Fabrication of thread-based patch for multiplexed sodium, ammonium, $\mathrm{pH}$, and lactate sensing. ${ }^{57}$ (d) Thread-based sensor for continuous perspiration level monitoring. ${ }^{58}$

\section{Future outlook and opportunities}

Thread, fibers, and textiles offer an astonishing opportunity to realize electronic, biomedical, and consumer electronic devices. They are naturally wearable and integrate seamlessly with the human body. Threads can be used as microfluidic channels, or coated with semiconducting inks to make transistors. They could be embedded into an organic tissue as a suture or assembled as a band-aid like patch for monitoring sweat and other biofluids. They provide a useful substrate to realize chemical and biological sensors, and in some cases, they can even be functionalized to change color in response to gases such as in 


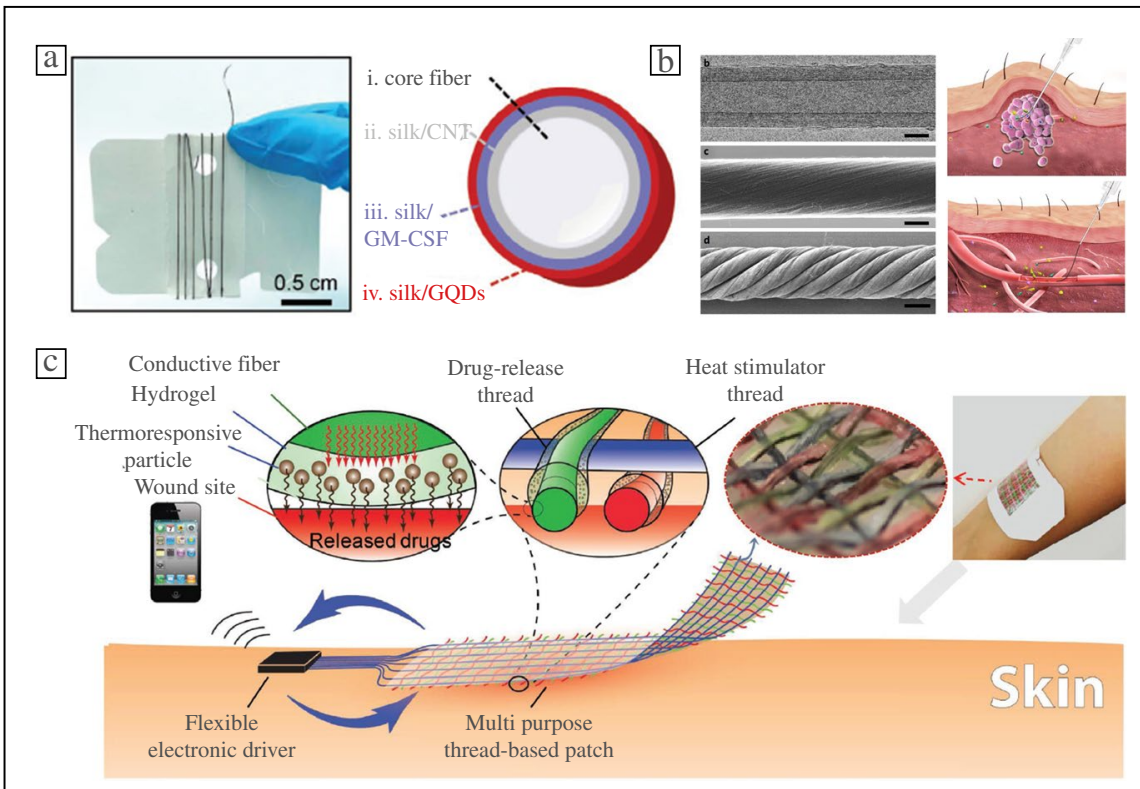

Figure 6. (a) Hierarchical structure of the biomimicking sensing suture. Reprinted with permission from Reference 60. (C) 2021 Wiley. (b) Hierarchical helical structure and implantation of the carbon nanotube-based sensing fiber. Reprinted with permission from Reference 61. () 2020 Springer Nature. (c) Multipurpose thread-based patch for transdermal drug delivery where drug release is triggered by heating conductive threads. Reproduced from Reference 62. @ 2017 Wiley. all kinds of biomarkers (e.g., metabolites and proteins) from different biological fluids such as sweat and urine. We can also envision clothing where threads connect together to form an intelligent system that monitors one's health and the environment, in real time, either for medical reasons where such information can save lives, or in consumer electronics application to enhance someone's experience such as a game controller for an immersive AR/VR gaming environment. In the context of innovations on threads and fiber as a material, newer material options and hybrids utilizing newer ways to synthesize them such as using electrospinning ${ }^{66,67}$ are expected to be promising since it allows one to control the material composition and the nanoscale features of the threads; these features will directly impact the quality and function of such threads. Given the hierarchical nature of threads and how they can be woven or embroidered into large fabrics, in multitude of patterns and configurapollution monitoring. While textiles have been around since the dawn of civilization, use of textile threads to make them smart so they can sense chemicals or detect physical activity is a newer scientific endeavor and is expected to grow. The one dimensional flexible nature of a thread provides a unique substrate that can be sewn or stitched onto any garment or can serve a standalone device itself. As we peer into the future, one can expect to see thread-based $\mu$ TAS to detect tions one can expect to achieve newer functionality by weaving different threads into a platform. A path to multifunctional sensors using multiple sensing threads is clearly possible. Recently, there also has been some work on threads that can deliver drugs on demand or serve as scaffolds for cell growth, topics out of the scope of this article, however, it foretells the future where one can integrate sensing and therapy in the same wearable platform using threads.

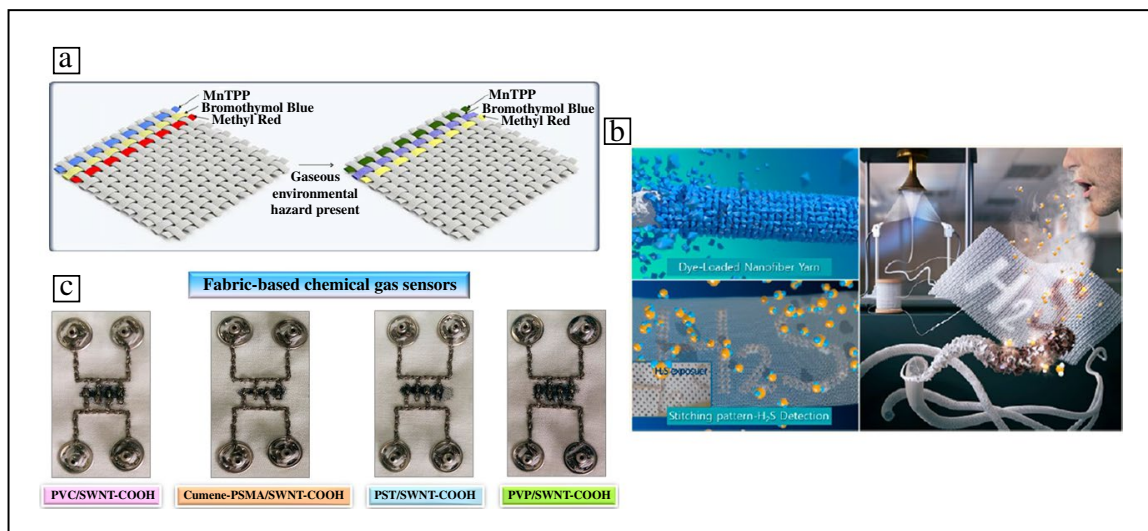

Figure 7. (a) Washable colorimetric gas sensing threads for detection of ammonium and hydrogen chloride. ${ }^{63}$ (b) Colorimetric dye-loaded electrospun nanofiber yarn for detection of hydrogen sulfide. Reprinted with permission from Reference 64. () 2020 American Chemical Society. (c) Bioinspired textile yarn using amyloid-graphene composite for detection of $\mathrm{NO}_{2}$. Reprinted with permission from Reference 54. () 2020 American Chemical Society.

\section{Acknowledgments}

This work is supported by the US Department of Defense (Award No. W81XWH-20-1-0589) and the National Science Foundation (Award No. 1931978).

\section{Funding}

Open access funding provided by Royal Institute of Technology.

\section{Conflict of interest}

On behalf of all authors, the corresponding author states that there is no conflict of interest. 


\section{Open Access}

This article is licensed under a Creative Commons Attribution 4.0 International License, which permits use, sharing, adaptation, distribution and reproduction in any medium or format, as long as you give appropriate credit to the original author(s) and the source, provide a link to the Creative Commons license, and indicate if changes were made. The images or other third party material in this article are included in the article's Creative Commons license, unless indicated otherwise in a credit line to the material. If material is not included in the article's Creative Commons license and your intended use is not permitted by statutory regulation or exceeds the permitted use, you will need to obtain permission directly from the copyright holder. To view a copy of this license, visit http://creativecommons.org/licenses/by/4.0/.

\section{References}

1. M.L. Hammock, A. Chortos, B.C.K. Tee, J.B.H. Tok, Z. Bao, 25th anniversary article: The evolution of electronic skin (E-Skin): A brief history, design considerations, and recent progress. Adv. Mater. 25(42), 5997 (2013)

2. D.H. Kim, N. Lu, R. Ma, Y.S. Kim, R.H. Kim, S. Wang, J. Wu, S.M. Won, H. Tao, A. Islam, K.J. Yu, T.I. Kim, R. Chowdhury, M. Ying, L. Xu, M. Li, H.J. Chung, H. Keum, M. McCormick, P. Liu, Y.W. Zhang, F.G. Omenetto, Y. Huang, T. Coleman, J.A. Rogers, Epidermal electronics. Science 333(6044), 838 (2011)

3. A.H. Najafabadi, A. Tamayol, N. Annabi, M. Ochoa, P. Mostafalu, M. Akbari, M. Nikkhah, R. Rahimi, M.R. Dokmeci, S. Sonkusale, B. Ziaie, A. Khademhosseini, Biodegradable nanofibrous polymeric substrates for generating elastic and flexible electronics. Adv. Mater. 26(33), 5823 (2014)

4. J.-Y. Sun, C. Keplinger, G.M. Whitesides, Z. Suo, Ionic skin. Adv. Mater. 26(45), 7608 (2014)

5. J.R. Windmiller, J. Wang, Wearable electrochemical sensors and biosensors: A review. Electroanalysis 25(1), 29 (2013)

6. T. Guinovart, G. Valdés-Ramírez, J.R. Windmiller, F.J. Andrade, J. Wang, Bandagebased wearable potentiometric sensor for monitoring wound $\mathrm{pH}$. Electroanalysis 26(6), 1345 (2014)

7. D.H. Kim, J. Song, W.M. Choi, H.S. Kim, R.H. Kim, Z. Liu, Y.Y. Huang, K.C. Hwang, Y.W. Zhang, J.A. Rogers, Materials and noncoplanar mesh designs for integrated circuits with linear elastic responses to extreme mechanical deformations. Proc. Nat. Acad. Sci. USA 105(48), 18675 (2008)

8. D.H. Kim, R. Ghaffari, N. Lu, S. Wang, S.P. Lee, H. Keum, R. D’Angelo, L. Klinker, Y. Su, C. Lu, Y.S. Kim, A. Ameen, Y. Li, Y. Zhang, B. de Graff, Y.Y. Hsu, Z. Liu, J. Ruskin, L. Xu, C. Lu, F.G. Omenetto, Y. Huang, M. Mansour, M.J. Slepian, J.A. Rogers, Electronic sensor and actuator webs for large-area complex geometry cardiac mapping and therapy. Proc. Nat. Acad. Sci. USA 109(49), 19910 (2012)

9. D.H. Kim, N. Lu, R. Ghaffari, Y.S. Kim, S.P. Lee, L. Xu, J. Wu, R.H. Kim, J. Song, Z. Liu, J. Viventi, B. de Graff, B. Elolampi, M. Mansour, M.J. Slepian, S. Hwang, J.D. Moss, S.M. Won, Y. Huang, B. Litt, J.A. Rogers, Materials for multifunctional balloon catheters with capabilities in cardiac electrophysiological mapping and ablation therapy. Nat. Mater. 10(4), 16 (2011)

10. P. Mostafalu, S. Sonkusale, A high-density nanowire electrode on paper for biomedical applications. RSC Adv. 5(12), 8680 (2015)

11. S. Xu, Y. Zhang, J. Cho, J. Lee, X. Huang, L. Jia, J.A. Fan, Y. Su, J. Su, H. Zhang, H. Cheng, B. Lu, C. Yu, C. Chuang, T.I. Kim, T. Song, K. Shigeta, S. Kang, C. Dagdeviren, I. Petrov, P.V. Braun, Y. Huang, U. Paik, J.A. Rogers, Stretchable batteries with self-similar serpentine interconnects and integrated wireless recharging systems. Nat. Commun. 4(1543), 2 (2013)

12. T. Yamada, Y. Hayamizu, Y. Yamamoto, Y. Yomogida, A. Izadi-Najafabadi, D.N. Futaba, K. Hata, A stretchable carbon nanotube strain sensor for human-motion detection. Nat. Nanotechnol. 6(5), 296 (2011)

13. J. Viventi, D.-H. Kim, L. Vigeland, E.S. Frechette, J.A. Blanco, Y.-S. Kim, A.E. Avrin, V.R. Tiruvadi, S.-W. Hwang, A.C. Vanleer, Flexible, foldable, actively multiplexed, highdensity electrode array for mapping brain activity in vivo. Nat. Neurosci. 14(12), 1599 (2011)

14. P. Mostafalu, S. Sonkusale, Flexible and transparent gastric battery: Energy harvesting from gastric acid for endoscopy application. Biosens. Bioelectron. 54, 292 (2014)
15. S.M. Sharaf, Smart Conductive Textile, in Advances in Functional and Protective Textiles, S. Ul-Islam and B.S. Butola, Eds. (Woodhead Publishing, 2020), pp. 141-167. 16. N. Belino, R. Fangueiro, S. Rana, P. Glampedaki, G. Priniotakis. Medical and healthcare textiles, in High Performance Technical Textiles, R. Paul, Ed., (Wiley, 2019), pp. 69 17. A. Lund, N.M. van der Velden, N.-K. Persson, M.M. Hamedi, C. Müller, Electrically conducting fibres for e-textiles: An open playground for conjugated polymers and carbon nanomaterials. Mater. Sci. Eng. R. Rep. 126, 1 (2018)

18. P. Mostafalu, M. Akbari, K.A. Alberti, Q. Xu, A. Khademhosseini, S.R. Sonkusale, A toolkit of thread-based microfluidics, sensors, and electronics for 3D tissue embedding for medical diagnostics. Microsyst. Nanoeng. 2, 16039 (2016)

19. M. Maccioni, E. Orgiu, P. Cosseddu, S. Locci, A. Bonfiglio, Towards the textile transistor: Assembly and characterization of an organic field effect transistor with a cylindrical geometry. Appl. Phys. Lett. 89(14), 43515 (2006)

20. A. Bonfiglio, D. De Rossi, T. Kirstein, I.R. Locher, F. Mameli, R. Paradiso, G. Vozzi Organic field effect transistors for textile applications. IEEE Trans. Inf. Technol. Biomed. 9(3), 319 (2005)

21. A. Babel, D. Li, Y. Xia, S.A. Jenekhe, Electrospun nanofibers of blends of conjugated polymers: Morphology, optical properties, and field-effect transistors. Macromolecules 38(11), 4705 (2005)

22. M. Hamedi, L. Herlogsson, X. Crispin, R. Marcilla, M. Berggren, O. Inganäs, Fiberembedded electrolyte-gated field-effect transistors for e-textiles. Adv. Mater. 21(5), 573 (2009)

23. M. Hamedi, R. Forchheimer, 0. Inganäs, Towards woven logic from organic electronic fibres. Nat. Mater. 6(5), 357 (2007)

24. R.E. Owyeung, T. Terse-Thakoor, H. Rezaei Nejad, M.J. Panzer, S.R. Sonkusale, Highly flexible transistor threads for all-thread based integrated circuits and multiplexed diagnostics. ACS Appl. Mater. Interfaces 11(4), 31096 (2019)

25. E. Battista, V. Lettera, M. Villani, D. Calestani, F. Gentile, P.A. Netti, S. lannotta, A Zappettini, N. Coppede, Enzymatic sensing with laccase-functionalized textile organic biosensors. Organ. Electron. 40, 51 (2017)

26. A. Yang, Y. Li, C. Yang, Y. Fu, N. Wang, L. Li, F. Yan, Fabric organic electrochemical transistors for biosensors. Adv. Mater. 30(23), 1800051 (2018)

27. R.L. McCreery, Advanced carbon electrode materials for molecular electrochemistry. Chem. Rev. 108(7), 2646(2008)

28. W. Ji, D. Wu, W. Tang, X. Xi, Y. Su, X. Guo, R. Liu, Carbonized silk fabric-based flexible organic electrochemical transistors for highly sensitive and selective dopamine detection. Sens. Actuators B 304, 127414 (2020)

29. Y. Zhang, Y. Wang, X. Qing, Y. Wang, W. Zhong, W. Wang, Y. Chen, Q. Liu, M. Li, D. Wang, Fiber organic electrochemical transistors based on multi-walled carbon nanotube and polypyrrole composites for noninvasive lactate sensing. Anal. Bioanal. Chem. 412(27), 7515 (2020)

30. Y. Wang, Z. Zhou, X. Qing, W. Zhong, Q. Liu, W. Wang, M. Li, K. Liu, D. Wang, Ion sensors based on novel fiber organic electrochemical transistors for lead ion detection. Anal. Bioanal. Chem. 408(21), 5779(2016)

31. X. Qing, Y. Wang, Y. Zhang, X. Ding, W. Zhong, D. Wang, W. Wang, Q. Liu, K. Liu, M. $\mathrm{Li}, \mathrm{Z}$. Lu, Wearable fiber-based organic electrochemical transistors as a platform for highly sensitive dopamine monitoring. ACS Appl. Mater. Interfaces 11(14), 13105 (2019) 32. T. Kumar, R.E. Owyeung, and S.R. Sonkusale, Rapid cleanroom-free fabrication of thread based transistors using three-dimensional stencil-based patterning. Flex. Print. Electron. 6(1), 015007 (2021)

33. jenaxinc.com. (accessed March 3, 2021).

34. J. Ren, Y. Zhang, W. Bai, X. Chen, Z. Zhang, X. Fang, W. Weng, Y. Wang, H. Peng, Elastic and wearable wire-shaped lithium-ion battery with high electrochemical performance. Angew. Chem. Int. Ed. 53(30), 7864 (2014)

35. F. Mo, G. Liang, Z. Huang, H. Li, D. Wang, C. Zhi, An overview of fiber-shaped batteries with a focus on multifunctionality, scalability, and technical difficulties. Adv. Mater. 32(5), e1902151 (2020)

36. A.S. Dahiya, J. Thireau, J. Boudaden, S. Lal, U. Gulzar, Y. Zhang, T. Gil, N. Azemard, P. Ramm, T. Kiessling, C. O'Murchu, F. Sebelius, J. Tilly, C. Glynn, S. Geary, C. O'Dwyer, K.M. Razeeb, A. Lacampagne, B. Charlot, A. Todri-Sanial, Review-energy autonomous wearable sensors for smart healthcare: A review. J. Electrochem. Soc. 167(3), 2 (2020) 37. Y. Cheng, R. Wang, J. Sun, L. Gao, A stretchable and highly sensitive graphenebased fiber for sensing tensile strain, bending, and torsion. Adv. Mater. 27(45), 7365 (2015)

38. A. Sadeqi, H.R. Nejad, F. Alaimo, H. Yun, M. Punjiya, S. Sonkusale, Washable smart threads for strain sensing fabrics. IEEE Sens. J. 25, 1 (2018)

39. S. Chen, Z. Lou, D. Chen, K. Jiang, G. Shen, Polymer-enhanced highly stretchable conductive fiber strain sensor used for electronic data gloves. Adv. Mater. Technol. 1(7), 15 (2016)

40. X. You, J. He, N. Nan, X. Sun, K. Qi, Y. Zhou, W. Shao, F. Liu, S. Cui, Stretchable capacitive fabric electronic skin woven by electrospun nanofiber coated yarns for detecting tactile and multimodal mechanical stimuli. J. Mater. Chem. C6(47), 12981 (2018)

41. F. Alaimo, A. Sadeqi, H. Rezaei Nejad, Y. Jiang, W. Wang, D. Demarchi, S. Sonkusale, Reel-to-reel fabrication of strain sensing threads and realization of smart insole. Sens. Actuators A 301, 54 (2020) 
42. N. Promphet, J.P. Hinestroza, P. Rattanawaleedirojn, N. Soatthiyanon, K. Siralertmukul, P. Potiyaraj, N. Rodthongkum, Cotton thread-based wearable sensor for noninvasive simultaneous diagnosis of diabetes and kidney failure. Sens. Actuators B 321, $128549(2020)$

43. R.S.P. Malon, L.Y. Heng, E.P. Córcoles, Recent developments in microfluidic paper-, cloth-, and thread-based electrochemical devices for analytical chemistry. Rev. Anal. Chem. 36(4), 1 (2017)

44. S. Farajikhah, J.M. Cabot, P.C. Innis, B. Paull, G. Wallace, Life-saving threads: Advances in textile-based analytical devices. ACS Comb. Sci. 21(4), 229 (2019)

45. B. Ma, J. Chi, C. Xu, Y. Ni, C. Zhao, H. Liu, Wearable capillary microfluidics for continuous perspiration sensing. Talanta 212, 120786 (2020)

46. Y.-E. Shin, J.-E. Lee, Y. Park, S.-H. Hwang, H.G. Chae, H. Ko, Sewing machine stitching of polyvinylidene fluoride fibers: Programmable textile patterns for wearable triboelectric sensors. J. Mater. Chem. A6(45), 22879 (2018)

47. I. ÖbergMånsson, A. Piper, M.M. Hamedi, Weaving off-the-shelf yarns into textile micro total analysis systems ( $\mu$ TAS). Macromol. Biosci. 20(11), 2000150 (2020)

48. Z. Abadi, V. Mottaghitalab, M. Bidoki, A. Benvidi, Flexible biosensor using inkjet printing of silver nanoparticles. Sens. Rev. 34(4), 360 (2014)

49. Y. Tajitsu, Development of e-textile sewn together with embroidered fabric having motion-sensing function using piezoelectric braided cord for embroidery. IEEE Trans. Dielectr. Electr. Insul. 27(5), 1644 (2020)

50. D.S. Kinnamon, S. Krishnan, S. Brosler, E. Sun, S. Prasad, Screen printed graphene oxide textile biosensor for applications in inexpensive and wearable point-of-exposure detection of influenza for at-risk populations. J. Electrochem. Soc. 165(8), B3084 (2018)

51. I. Gualandi, M. Marzocchi, A. Achilli, D. Cavedale, A. Bonfiglio, B. Fraboni, Textile organic electrochemical transistors as a platform for wearable biosensors. Sci. Rep. 6(1), 1 (2016)

52. R. Rahimi, W. Yu, T. Parupudi, M. Ochoa, B. Ziaie, A Low-Cost Fabrication Technique for Direct Sewing Stretchable Interconnetions for Wearable Electronics, in 2015 Transducers-2015 18th International Conference on Solid-State Sensors, Actuators and Microsystems (TRANSDUCERS) (IEEE, 2015) pp. 1350-1353

53. X. Liu, P.B. Lillehoj, Embroidered electrochemical sensors for biomolecular detection. Lab Chip 16(11), 2093 (2016)

54. T. Seesaard, P. Lorwongtragool, T. Kerdcharoen, Development of fabric-based chemical gas sensors for use as wearable electronic noses. Sensors 15(1), 1885 (2015)

55. A. Modali, S.R.K Vanjari, D. Dendukuri, Wearable woven electrochemical biosensor patch for non-invasive diagnostics. Electroanalysis 28(6), 1276 (2016)

56. T. Choudhary, G.P. Rajamanickam, D. Dendukuri, Woven electrochemical fabricbased test sensors (WEFTS): A new class of multiplexed electrochemical sensors. Lab Chip 15(9), 2064 (2015)

57. T. Terse-Thakoor, M. Punjiya, Z. Matharu, B. Lyu, M. Ahmad, G.E. Giles, R. Owyeung, F. Alaimo, M. Shojaei-Baghini, T.T. Brunyé, S. Sonkusale, Thread-based multiplexed sensor patch for real-time sweat monitoring. npj Flex. Electron. 4(1), 20 (2020) 58. J. Jia, C. Xu, S. Pan, S. Xia, P. Wei, H.Y. Noh, P. Zhang, X. Jiang, Conductive threadbased textile sensor for continuous perspiration level monitoring. Sensors (Basel) 18(11), 15 (2018)

59. A. Lendlein, R. Langer, Biodegradable, elastic shape-memory polymers for potential biomedical applications. Science 296(31), 1673 (2002)

60. M. Liu, Y. Zhang, K. Liu, G. Zhang, Y. Mao, L. Chen, Y. Peng, T.H. Tao, Biomimicking antibacterial opto-electro sensing sutures made of regenerated silk proteins. Adv. Mater. 33(1), 2004733 (2021)

61. L. Wang, S. Xie, Z. Wang, F. Liu, Y. Yang, C. Tang, X. Wu, P. Liu, Y. Li, H. Saiyin, S. Zheng, X. Sun, F. Xu, H. Yu, H. Peng, Functionalized helical fibre bundles of carbon nanotubes as electrochemical sensors for long-term in vivo monitoring of multiple disease biomarkers. Nat. Biomed. Eng. 4(2), 159 (2020)

62. P. Mostafalu, G. Kiaee, G. Giatsidis, A. Khalilpour, M. Nabavinia, M.R. Dokmeci, S. Sonkusale, D.P. Orgill, A. Tamayol, A. Khademhosseini, A textile dressing for temporal and dosage controlled drug delivery. Adv. Func. Mater. 27(41), 1702399 (2017)

63. R.E. Owyeung, M.J. Panzer, S.R. Sonkusale, Colorimetric gas sensing washable threads for smart textiles. Sci. Rep. 9(1), 5607 (2019)

64. D.H. Kim, J.H. Cha, J.Y. Lim, J. Bae, W. Lee, K.R. Yoon, C. Kim, J.S. Jang, W. Hwang, I.D. Kim, Colorimetric dye-loaded nanofiber yarn: Eye-readable and weavable gas sensing platform. ACS Nano 14(12), 16907 (2020)

65. M.J. Chan, Y.J. Li, C.C. Wu, Y.C. Lee, H.W. Zan, H.F. Meng, M.H. Hsieh, C.S. Lai, Y.C. Tian, Breath ammonia is a useful biomarker predicting kidney function in chronic kidney disease patients. Biomedicines 8(11), 31 (2020)

66. M.N. Shuakat, T. Lin, Recent developments in electrospinning of nanofiber yarns. J. Nanosci. Nanotechnol. 14(2), 1389 (2014)

67. A. Mouthuy, N. Zargar, 0. Hakimi, E. Lostis, A. Carr, Fabrication of continuous electrospun filaments with potential for use as medical fibres. Biofabrication 7(2) 025006 (2015)

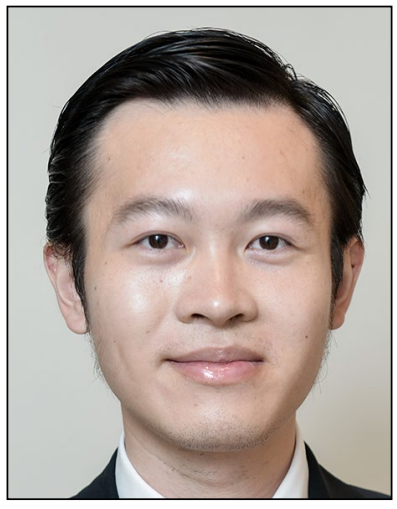

Junfei Xia is a postdoctoral research associate in the Department of Electrical and Computer Engineering at Tufts University. He received his BS degree in biotechnology from China Pharmaceutical University, China, in 2011, and his $\mathrm{PhD}$ degree in biomedical engineering from Florida State University in 2016. He conducted postdoctoral research at Northeastern University from 2016 to 2019, where he studied nanosensors for in vivo imaging. Xia joined the Nano Lab at Tufts University in 2019 to work on flexible textile-based biosensors. His research interests include biosensors, lab-on-a-chip devices, biomaterials, and micro-/nanodevices for drug delivery. Xia can be reached by email at junfei.xia@tufts.edu.

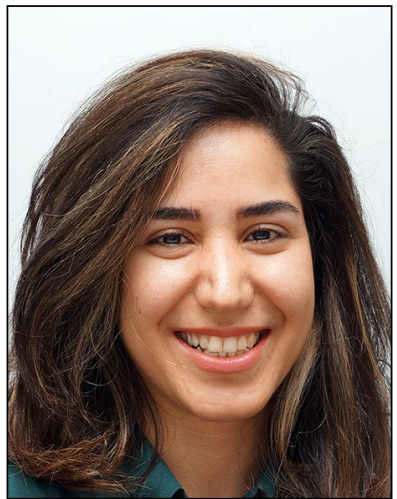

Shirin Khaliliazar received her BS and MSc degrees in textile engineeringfiber science and technology from the Tehran Polytechnic (Amirkabir University of Technology), Iran, in 2008 and 2014 . She is currently conducting doctoral research at the KTH Royal Institute of Technology, Sweden. Her research focuses on fiber-based diagnostic devices for infectious diseases in point-of-care applications. Her research interests include electroanalytical biosensors, point-of-care molecular diagnostics, and biomaterials for drug delivery and medical applications. Khaliliazar can be reached by email at shirink@kth.se.



Mahiar Max Hamedi is a professor at the school of Engineering Sciences in Chemistry, Biotechnology and Health, at the Royal Institute of Technology (KTH), Sweden. He received his $\mathrm{PhD}$ degree in organic electronics from Linköping University, Sweden, and was a visiting researcher at Harvard University. His research has been highlighted by many news organizations, including BBC, Science, Nature, CNN and the MIT Tech. Review. He has received several awards, including the European Research Council Award. Hamedi can be reached by email at mahiar@kth.se.

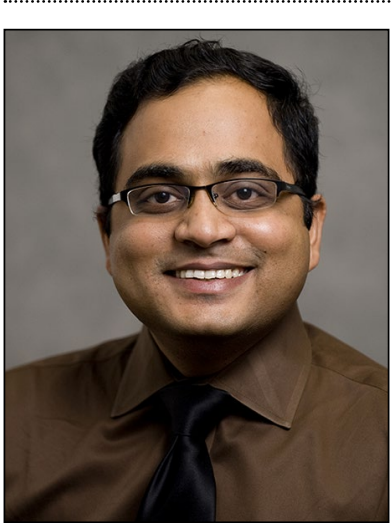

Sameer Sonkusale is a professor of electrical and computer engineering, biomedical engineering, and chemical and biological engineering at Tufts University. He was a visiting faculty at Harvard Medical School and the Wyss Institute. He received his MS and PhD degrees in electrical engineering from the University of Pennsylvania. His research interests include flexible bioelectronics, biomedical devices circuits and systems, and diagnostics. He has received several awards, including the CAREER Award from the National Science Foundation. Sonkusale can be reached by email at sameer@ece. tufts.edu. 